\title{
ЭКСТРЕМАЛЬНАЯ ИНТЕРПОЛЯЦИЯ НА ПОЛУОСИ С НАИМЕНЬШИМ ЗНАЧЕНИЕМ НОРМЫ ТРЕТЬЕЙ ПРОИЗВОДНОЙ ${ }^{1}$
}

\author{
С. И. Новиков, В. Т. Шевалдин
}

В работе рассмотрена следующая задача. Для класса интерполируемых последовательностей $y=\left\{y_{k}\right\}_{k=-\infty}^{+\infty}$ действительных чисел, у которых разделенные разности третьего порядка, построенные по произвольным узлам $\left\{x_{k}\right\}_{k=-\infty}^{+\infty}$, ограничены по модулю фиксированным положительным числом, на классе функций, имеющих почти всюду третью производную, требуется найти функцию $f$ такую, что $f\left(x_{k}\right)=y_{k}(k \in \mathbb{Z})$, и третья производная которой имеет наименьшую $L_{\infty}$-норму. В работе получено решение этой задачи на положительной полуоси $\mathbb{R}_{+}=(0,+\infty)$ для геометрических сеток, последовательность шагов которых $h_{k}=x_{k+1}-x_{k}(k \in \mathbb{Z})$ образует геометрическую прогрессию со знаменателем $p(p>1)$, т. е. $h_{k+1} / h_{k}=p$. В случае равномерной сетки $x_{k}=k h(h>0, k \in \mathbb{Z})$ на всей оси $\mathbb{R}($ т. е. при $p=1)$ эта задача была решена Ю. Н. Субботиным в 1965 году и известна как задача Яненко - Стечкина - Субботина экстремальной функциональной интерполяции.

Ключевые слова: интерполяция, разделенная разность, сплайны, разностное уравнение.

S. I. Novikov, V.T. Shevaldin. Extremal interpolation on the semiaxis with the smallest norm of the third derivative.

The following problem is considered. For a class of interpolated sequences $y=\left\{y_{k}\right\}_{k=-\infty}^{+\infty}$ of real numbers such that their third-order divided difference constructed for arbitrary knots $\left\{x_{k}\right\}_{k=-\infty}^{+\infty}$ are bounded in absolute value by a fixed positive number, it is required to find a function $f$ having the third derivative almost everywhere and such that $f\left(x_{k}\right)=y_{k}(k \in \mathbb{Z})$ and the third derivative has the smallest $L_{\infty}$-norm. The problem is solved on the positive semiaxis $\mathbb{R}_{+}=(0,+\infty)$ for geometric grids in which the sequence of steps $h_{k}=x_{k+1}-x_{k}$ $(k \in \mathbb{Z})$ is a geometric progression with ratio $p(p>1)$; i.e., $h_{k+1} / h_{k}=p$. In the case of a uniform grid $x_{k}=k h(h>0, k \in \mathbb{Z})$ on the whole axis $\mathbb{R}$ (i.e., for $p=1$ ), this problem was solved by Yu. N. Subbotin in 1965 and is known as the Yanenko-Stechkin-Subbotin problem of extremal function interpolation.

Keywords: interpolation, divided difference, splines, difference equation.

MSC: $41 \mathrm{~A} 15$

DOI: $10.21538 / 0134-4889-2020-26-4-210-223$

\section{1. Введение}

Пусть $n$ - произвольное натуральное число и на числовой оси $\mathbb{R}$ задана сетка узлов $\Delta=\left\{x_{k}\right\}_{k=-\infty}^{+\infty}$ вида

$$
\mathfrak{a}<\cdots<x_{k}<x_{k+1}<x_{k+2}<\cdots<\mathfrak{b},
$$

где $\mathfrak{a}=\inf _{k} x_{k}, \mathfrak{b}=\sup _{k} x_{k}$. Здесь $\mathfrak{a}-$ число или $\mathfrak{a}=-\infty$ и аналогично $\mathfrak{b}-$ число или $\mathfrak{b}=+\infty$. Величины $h_{k}=x_{k+1}-x_{k}(k \in \mathbb{Z})$ будем называть шагами сетки (1.1).

Для функции $f:(\mathfrak{a}, \mathfrak{b}) \rightarrow \mathbb{R}$ полагаем $f\left(x_{k}\right)=y_{k}(k \in \mathbb{Z})$, где $y=\left\{y_{k}\right\}_{k=-\infty}^{+\infty}-$ произвольная последовательность действительных чисел. Как известно (см., например, [1, гл. 1]), разделенные разности произвольного порядка $n$ от функции $f$ на сетке (1.1) определяются рекуррентно при помощи равенств

$$
\begin{gathered}
f\left[x_{k+1}, x_{k}\right]=\left[y_{k+1}, y_{k}\right]=\frac{y_{k+1}-y_{k}}{x_{k+1}-x_{k}}, \\
f\left[x_{k+2}, x_{k+1}, x_{k}\right]=\left[y_{k+2}, y_{k+1}, y_{k}\right]=\frac{\left[y_{k+2}, y_{k+1}\right]-\left[y_{k+1}, y_{k}\right]}{x_{k+2}-x_{k}},
\end{gathered}
$$

\footnotetext{
${ }^{1}$ Работа выполнена в рамках исследований, проводимых в Уральском математическом центре.
} 


$$
f\left[x_{k+n}, \ldots, x_{k}\right]=\left[y_{k+n}, \ldots, y_{k}\right]=\frac{\left[y_{k+n}, \ldots, y_{k+1}\right]-\left[y_{k+n-1}, \ldots, y_{k}\right]}{x_{k+n}-x_{k}} .
$$

В [1] отмечены основные свойства разделенных разностей (в частности, обращение в нуль на многочленах степени $\leq n-1)$ и приведены другие формы их представления. Если сетка точек интерполяции (1.1) является равномерной, то разделенная разность $n$-го порядка совпадает с точностью до постоянного положительного множителя с конечной разностью порядка $n$ $\Delta_{h}^{n} y_{k}=\sum_{\nu=0}^{n}(-1)^{n-\nu} C_{n}^{\nu} y_{k+\nu}$, а именно, имеет место равенство

$$
f\left[x_{k+n}, \ldots, x_{k}\right]=\frac{1}{h^{n} n !} \Delta_{h}^{n} f\left(x_{k}\right) .
$$

Класс интерполируемых последовательностей, который мы рассматриваем в настоящей работе, задается следующим образом:

$$
Y_{n}=\left\{y=\left\{y_{k}\right\}_{k=-\infty}^{\infty}: \sup _{k \in \mathbb{Z}}\left|\left[y_{k+n}, y_{k+n-1}, \ldots, y_{k}\right]\right| \leq 1\right\} .
$$

Для любой последовательности $y \in Y_{n}$ рассмотрим класс функций

$$
F_{n}(y)=\left\{f: f^{(n-1)} \in A C, f^{(n)} \in L_{\infty}(\mathfrak{a} ; \mathfrak{b}), f\left(x_{k}\right)=y_{k} \quad(k \in \mathbb{Z})\right\} .
$$

Здесь, как обычно, $A C$ - множество локально абсолютно непрерывных функций, а $L_{\infty}(\mathfrak{a}, \mathfrak{b})-$ класс функций, существенно ограниченных на интервале $(\mathfrak{a}, \mathfrak{b})$ с обычным определением равномерной нормы

$$
\|f\|_{\infty}=\|f\|_{L_{\infty}(\mathfrak{a}, \mathfrak{b})}=\operatorname{ess}_{x \in(\mathfrak{a}, \mathfrak{b})}|f(x)| .
$$

Задача экстремальной функииональной интерполяиии заключается в точном вычислении (или получении эффективных оценок сверху и снизу) величины

$$
A_{n}(\Delta)=\sup _{y \in Y_{n}} \inf _{f \in F_{n}(y)}\left\|f^{(n)}\right\|_{\infty}
$$

Другими словами, задача (1.3) состоит в том, чтобы найти наименьшую норму $n$-й производной интерполирующей функции из класса $F_{n}(y)$ для "наихудшей" интерполируемой последовательности $y \in Y_{n}$.

Хорошо известно (см., например, [1, с. 40]) следующее свойство разделенных разностей: если $n$-я производная некоторой действительной функции $f(x)$ ограничена сверху по модулю некоторой положительной константой $M$ для всех $x \in(\mathfrak{a}, \mathfrak{b})$, то абсолютная величина разделенной разности порядка $n$ на любой сетке узлов $\Delta$ из промежутка $(\mathfrak{a}, \mathfrak{b})$ не превосходит числа $M / n$ !. Задачу (1.3) можно считать обратной к этому свойству разделенных разностей.

Задача (1.3) по постановке близка к известной интерполяционной проблеме Фавара [2], в которой на последовательность разделенных разностей накладывалось лишь конечное число ограничений.

Для равномерной сетки точек интерполяции (т. е. для конечных разностей $n$-го порядка) постановка задачи (1.3) принадлежит С. Б. Стечкину (а обратил его внимание на эту задачу Н. Н. Яненко, занимавшийся разностными методами решения дифференциальных уравнений). Ю.Н. Субботин в работе [3] нашел точное решение этой задачи. В дальнейшем подобные задачи были рассмотрены им в пространствах $L_{q}$ при $1 \leq q<\infty$ (см. [4]), а также возникли другие многочисленные обобщения и применения теории, развитой в работах Ю. Н. Субботина. Подробный обзор результатов этих исследований содержится в работе авторов 2018 г. (Субботин Ю.Н., Новиков С. И., Шевалдин В. Т. Экстремальная функциональная интерполяция и сплайны // Тр. Ин-та математики и механики УрО РАН. 2018. Т. 24, № 3. С. 200-225). На указанную работу будем ссылаться ниже как на обзор. 
Для сеток, не являющихся равномерными, задача (1.3) является более трудной. Случай $n=1$ сводится к локальной интерполяции ломаными и потому не представляет интереса. Случай $n=2$ рассмотрен в недавней статье авторов (Новиков С. И., Шевалдин В. Т. О связи между второй разделенной разностью и второй производной // Тр. Института математики и механики УрО РАН. 2020. Т. 26, № 2. С. 216-224). Отметим также, что оценки производных интерполирующих функций через разделенные разности интерполируемых значений можно найти в работе [5] и приведенной там литературе.

В настоящей работе мы рассматриваем случай $n=3$ и ограничиваемся интерполяцией на геометрических сетках узлов $\Delta_{p}$. Это сетки вида (1.1), последовательность шагов $\left\{h_{k}\right\}$ которых образует геометрическую прогрессию со знаменателем $p>1$, т. е.

$$
\frac{h_{k+1}}{h_{k}}=p \quad(k \in \mathbb{Z})
$$

При $p=1$ геометрическая прогрессия переходит в арифметическую, и мы имеем дело с равномерной сеткой.

Цель настоящей работы - найти точное значение величины $A_{3}\left(\Delta_{p}\right)$ при $p>1$. Основным результатом является следующая

Теорема 1. При $p \geq 1$ справедливо равенство

$$
A_{3}\left(\Delta_{p}\right)=\frac{6\left(p^{2}+p+1\right)}{\left(p^{2}-p+1\right)} \frac{\left(p^{2}+1\right)^{2}}{(p-1)^{3}(p+1)+4 p \sqrt{p\left(p^{2}-p+1\right)}} .
$$

Для равномерной сетки узлов интерполяции на всей вещественной оси Ю. Н. Субботин [3] доказал, что $A_{3}\left(\Delta_{1}\right)=(3 !) \cdot 3=18$ (см. (1.2) при $\left.n=3\right)$. Это число совпадает с правой частью равенства в теореме 1 при $p=1$.

Наше доказательство теоремы 1 базируется на подходе Ю. Н. Субботина [3], однако реализация этого подхода с учетом специфики неравномерной геометрической сетки узлов интерполяции на полуоси требует дополнительных исследований.

В первом разделе настоящей работы получено представление разделенной разности третьего порядка функции $f$, заданной своими значениями в точках геометрической сетки, в виде суммы интегралов, и с его помощью найдена оценка снизу $L_{\infty}$-нормы третьей производной произвольного интерполянта из класса $F_{3}\left(y^{*}\right)$ для некоторой, выбранной специальным образом последовательности интерполируемых данных $y^{*} \in Y_{3}$. Во втором разделе для произвольной последовательности интерполируемых данных $y \in Y_{3}$ получена оценка сверху $L_{\infty}$-нормы третьей производной интерполирующей функции и доказана теорема 1.

При этом экстремальными функциями для величины $A_{3}\left(\Delta_{p}\right)$ являются интерполирующие на исходной сетке кубические сплайны с неравномерными узлами "склейки" в найденных нами точках $\left\{t_{k}\right\}_{k=-\infty}^{+\infty}$, которые расположены между узлами интерполяции, а экстремальной последовательностью $y^{*}=\left\{y_{k}^{*}\right\}_{k=-\infty}^{+\infty} \in Y_{3}$ интерполируемых данных - та, разделенные разности которой равны по модулю единице и чередуют знаки, т. е. $\left[y_{k+3}^{*}, y_{k+2}^{*}, y_{k+1}^{*}, y_{k}^{*}\right]=(-1)^{k}, k \in \mathbb{Z}$.

\section{2. Представление разделенной разности третьего порядка и оценка снизу нормы интерполирующей функции}

Пусть $p>1$. Для геометрической сетки $\Delta_{p}$ можно, не ограничивая общности, считать, что ее узлы имеют вид

$$
x_{k}=p^{k} h,
$$

где $h>0$ - некоторая константа, $k \in \mathbb{Z}$. Действительно, условия $h_{k+1} / h_{k}=p$ равносильны разностному уравнению $x_{k+2}-(p+1) x_{k+1}+p x_{k}=0$, решая которое, получаем $x_{k}=p^{k} h+\gamma(k \in \mathbb{Z})$ и замечаем, что величина $\gamma$ (не зависящая от $k$ ) не влияет на значения разделенных разностей. 
Поэтому полагаем $\gamma=0$. Вид узлов $(2.1)$ означает, что они расположены на положительной полуоси $\mathbb{R}_{+}=(0,+\infty)$, т. е. в определении сетки (1.1) имеем $\mathfrak{a}=0, \mathfrak{b}=+\infty$.

Получим интегральное представление разделенной разности третьего порядка в удобной для нас форме.

Лемма 1. Для любой функиии $f \in F_{3}(y)$ при $p>1$ справедливо представление

$$
\begin{gathered}
f\left[x_{k+3}, x_{k+2}, x_{k+1}, x_{k}\right]=\frac{1}{2 p^{3(k+1)}(p-1)\left(p^{2}-1\right)\left(p^{3}-1\right) h^{3}} \\
\times\left(\int_{x_{k}}^{x_{k+3}}\left(x_{k+3}-t\right)^{2} f^{\prime \prime \prime}(t) d t-\left(1+p+p^{2}\right) \int_{x_{k}}^{x_{k+2}}\left(x_{k+2}-t\right)^{2} f^{\prime \prime \prime}(t) d t\right. \\
\left.+p\left(1+p+p^{2}\right) \int_{x_{k}}^{x_{k+1}}\left(x_{k+1}-t\right)^{2} f^{\prime \prime \prime}(t) d t\right) .
\end{gathered}
$$

Д о к а з а т е л ь с т в о. Точки (2.1) подставляем в формулы, последовательно определяющие разделенные разности третьего порядка. В результате имеем

$$
\begin{gathered}
{\left[y_{k+1}, y_{k}\right]=\frac{y_{k+1}-y_{k}}{p^{k}(p-1) h}, \quad\left[y_{k+2}, y_{k+1}, y_{k}\right]=\frac{y_{k+2}-(p+1) y_{k+1}+p y_{k}}{p^{2 k+1}(p-1)\left(p^{2}-1\right) h^{2}},} \\
{\left[y_{k+3}, y_{k+2}, y_{k+1}, y_{k}\right]=\frac{y_{k+3}-\left(1+p+p^{2}\right) y_{k+2}+\left(p^{3}+p^{2}+p\right) y_{k+1}-p^{3} y_{k}}{p^{3(k+1)}(p-1)\left(p^{2}-1\right)\left(p^{3}-1\right) h^{3}} .}
\end{gathered}
$$

Функцию $f \in F_{3}(y)$ записываем с помощью формулы Тейлора с остаточным членом в интегральной форме

$$
f(x)=f\left(x_{k}\right)+f^{\prime}\left(x_{k}\right)\left(x-x_{k}\right)+\frac{f^{\prime \prime}\left(x_{k}\right)}{2 !}\left(x-x_{k}\right)^{2}+\frac{1}{2} \int_{x_{k}}^{x}(x-t)^{2} f^{\prime \prime \prime}(t) d t .
$$

Поскольку все внеинтегральные слагаемые уничтожаются в силу того, что разделенная разность третьего порядка обращается в нуль на квадратичных функциях, отсюда получаем представление (2.2). Лемма 1 доказана.

Утверждение 1. Для последовательности $y^{*}=\left\{y_{k}^{*}\right\}_{k=-\infty}^{+\infty} \in Y_{3}$, удовлетворяющей условию

$$
\left[y_{k+3}^{*}, y_{k+2}^{*}, y_{k+1}^{*}, y_{k}^{*}\right]=(-1)^{k} \quad(k \in \mathbb{Z}),
$$

и любой функии $f \in F_{3}\left(y^{*}\right)$ при всех $p>1$ выполняется неравенство

$$
\sup _{x \in \mathbb{R}_{+}}\left|f^{\prime \prime \prime}(x)\right| \geq \frac{6\left(p^{2}+p+1\right)}{\left(p^{2}-p+1\right)} \frac{\left(p^{2}+1\right)^{2}}{(p-1)^{3}(p+1)+4 p \sqrt{p\left(p^{2}-p+1\right)}} .
$$

Д о к а з а т е л ь с т в о. Пусть последовательность $y^{*}$ удовлетворяет условию (2.3) и $f \in F_{3}\left(y^{*}\right)$. Из леммы 1 после преобразования интегралов имеем

$$
\begin{gathered}
f\left[x_{k+3}, x_{k+2}, x_{k+1}, x_{k}\right]=\frac{1}{2 p^{3(k+1)}(p-1)\left(p^{2}-1\right)\left(p^{3}-1\right) h^{3}} \\
\times\left(\int_{x_{k+2}}^{x_{k+3}}\left(x_{k+3}-t\right)^{2} f^{\prime \prime \prime}(t) d t+\int_{x_{k+1}}^{x_{k+2}}\left[\left(x_{k+3}-t\right)^{2}-\left(1+p+p^{2}\right)\left(x_{k+2}-t\right)^{2}\right] f^{\prime \prime \prime}(t) d t\right.
\end{gathered}
$$




$$
\left.+\int_{x_{k}}^{x_{k+1}}\left[\left(x_{k+3}-t\right)^{2}-\left(1+p+p^{2}\right)\left(x_{k+2}-t\right)^{2}+p\left(1+p+p^{2}\right)\left(x_{k+1}-t\right)^{2}\right] f^{\prime \prime \prime}(t) d t\right) .
$$

В первом интеграле делаем замену переменной интегрирования, полагая $t=z h^{-1}\left(x_{k+3}-\right.$ $\left.x_{k+2}\right)+x_{k+2}$, во втором $-t=z h^{-1}\left(x_{k+2}-x_{k+1}\right)+x_{k+1}$ и в третьем $-t=z h^{-1}\left(x_{k+1}-x_{k}\right)+x_{k}$. Тем самым каждый из них сводится к интегралу по промежутку $[0, h]$ и после выполнения элементарных преобразований получаем

$$
\begin{gathered}
{\left[y_{k+3}^{*}, y_{k+2}^{*}, y_{k+1}^{*}, y_{k}^{*}\right]=\frac{1}{2 h^{3}(p+1)\left(p^{2}+p+1\right)}\left(p^{3} \int_{0}^{h}(h-z)^{2} f^{\prime \prime \prime}\left(\left(p^{k+3}-p^{k+2}\right) z+p^{k+2} h\right) d z\right.} \\
\left.+p \int_{0}^{h}\left(h^{2}+2 p h z-(1+p) z^{2}\right) f^{\prime \prime \prime}\left(\left(p^{k+2}-p^{k+1}\right) z+p^{k+1} h\right) d z+\int_{0}^{h} z^{2} f^{\prime \prime \prime}\left(\left(p^{k+1}-p^{k}\right) z+p^{k} h\right) d z\right) .
\end{gathered}
$$

Пусть $N$ - натуральное число, $N \geq 3$. Из (2.4) и (2.3) имеем

$$
\begin{gathered}
2 N+1=\sum_{k=-N}^{N}(-1)^{k}\left[y_{k+3}^{*}, y_{k+2}^{*}, y_{k+1}^{*}, y_{k}^{*}\right] \\
=\mu \sum_{k=-N}^{N}(-1)^{k}\left(p^{3} \int_{0}^{h}(h-z)^{2} f^{\prime \prime \prime}\left(\left(p^{k+3}-p^{k+2}\right) z+p^{k+2} h\right) d z\right.
\end{gathered}
$$

$\left.+p \int_{0}^{h}\left(h^{2}+2 p h z-(1+p) z^{2}\right) f^{\prime \prime \prime}\left(\left(p^{k+2}-p^{k+1}\right) z+p^{k+1} h\right) d z+\int_{0}^{h} z^{2} f^{\prime \prime \prime}\left(\left(p^{k+1}-p^{k}\right) z+p^{k} h\right) d z\right)=S_{1}+S_{2}$, где $\mu=\left(2 h^{3}(p+1)\left(p^{2}+p+1\right)\right)^{-1}$ и

$$
\begin{aligned}
S_{1} & =\mu\left(p^{3}(-1)^{N} \int_{0}^{h}(h-z)^{2} f^{\prime \prime \prime}\left(\left(p^{N+3}-p^{N+2}\right) z+p^{N+2} h\right) d z\right. \\
+p(-1)^{N} \int_{0}^{h}\left(h^{2}+2 p h z-(1+p) z^{2}\right) f^{\prime \prime \prime}\left(\left(p^{N+2}-p^{N+1}\right) z+p^{N+1} h\right) d z & \\
+ & (-1)^{N-1} p^{3} \int_{0}^{h}(h-z)^{2} f^{\prime \prime \prime}\left(\left(p^{N+2}-p^{N+1}\right) z+p^{N+1} h\right) d z \\
+ & (-1)^{-N+1} \int_{0}^{h} z^{2} f^{\prime \prime \prime}\left(\left(p^{-N+2}-p^{-N+1}\right) z+p^{-N+1} h\right) d z \\
+p(-1)^{-N} & \int_{0}^{h}\left(h^{2}+2 p h z-(1+p) z^{2}\right) f^{\prime \prime \prime}\left(\left(p^{-N+2}-p^{-N+1}\right) z+p^{-N+1} h\right) d z \\
& \left.+(-1)^{-N} \int_{0}^{h} z^{2} f^{\prime \prime \prime}\left(\left(p^{-N+1}-p^{-N}\right) z+p^{-N} h\right) d z\right)
\end{aligned}
$$




$$
S_{2}=\mu(p+1) \int_{0}^{h}\left(z^{2}\left(p^{2}+1\right)-2 h p^{2} z+p h^{2}(p-1)\right) \sum_{k=-N+2}^{N}(-1)^{k} f^{\prime \prime \prime}\left(\left(p^{k+1}-p^{k}\right) z+p^{k} h\right) d z .
$$

Теперь оцениваем сверху величины $\left|S_{1}\right|$ и $\left|S_{2}\right|$.

$$
\begin{gathered}
\left|S_{1}\right| \leq 2 \mu\left(p^{3} \int_{0}^{h}(h-z)^{2} d z+p \int_{0}^{h}\left|h^{2}+2 p h z-(1+p) z^{2}\right| d z+\int_{0}^{h} z^{2} d z\right) \sup _{x \in \mathbb{R}_{+}}\left|f^{\prime \prime \prime}(x)\right| \\
=\frac{2 \mu}{3} h^{3}(p+1)\left(p^{2}+p+1\right)\left(\sup _{x \in \mathbb{R}_{+}}\left|f^{\prime \prime \prime}(x)\right|\right)=\frac{1}{3} \sup _{x \in \mathbb{R}_{+}}\left|f^{\prime \prime \prime}(x)\right| .
\end{gathered}
$$

Для того чтобы получить оценку величины $\left|S_{2}\right|$, определяем функцию

$$
g(z)=z^{2}\left(p^{2}+1\right)-2 h p^{2} z+p h^{2}(p-1), \quad z \in[0, h] .
$$

Относительно $z$ эта функция является квадратным трехчленом и имеет единственный нуль на $[0, h)$ в точке

$$
z_{0}=z_{0}(p)=h\left(\frac{p^{2}-\sqrt{p\left(p^{2}-p+1\right)}}{1+p^{2}}\right) .
$$

Замечаем, что $z_{0}(1)=0$, а при $p>1$ имеем $z_{0}(p) \in(0, h)$, и функция $g(z)$ при переходе через точку $z_{0}(p)$ меняет знак с плюса на минус. Поэтому

$$
\begin{gathered}
\left|S_{2}\right| \leq \mu(2 N-1)(p+1)\left(\sup _{x \in \mathbb{R}_{+}}\left|f^{\prime \prime \prime}(x)\right|\right) \int_{0}^{h}|g(z)| d z \\
=\mu(2 N-1)(p+1)\left(\sup _{x \in \mathbb{R}_{+}}\left|f^{\prime \prime \prime}(x)\right|\right)\left(\int_{0}^{z_{0}} g(z) d z-\int_{z_{0}}^{h} g(z) d z\right) .
\end{gathered}
$$

Таким образом, из неравенства $2 N+1 \leq\left|S_{1}\right|+\left|S_{2}\right|$ получаем

$$
\sup _{x \in \mathbb{R}_{+}}\left|f^{\prime \prime \prime}(x)\right| \geq \frac{2 N+1}{1 / 3+\mu(2 N-1)(p+1)\left(\int_{0}^{z_{0}} g(z) d z-\int_{z_{0}}^{h} g(z) d z\right)} .
$$

В этом неравенстве переходим к пределу при $N \rightarrow \infty$ и с учетом определения функции $g(z)$ и выражений для $\mu$ и $z_{0}$ после вычисления интегралов и выполнения элементарных преобразований приходим к оценке

$$
\sup _{x \in \mathbb{R}_{+}}\left|f^{\prime \prime \prime}(x)\right| \geq \frac{6\left(p^{2}+p+1\right)}{\left(p^{2}-p+1\right)} \frac{\left(p^{2}+1\right)^{2}}{(p-1)^{3}(p+1)+4 p \sqrt{p\left(p^{2}-p+1\right)}} .
$$

Утверждение 1 доказано.

\section{3. Оценка нормы третьей производной интерполирующей функции и доказательство теоремы 1}

Построим функцию $\tilde{f}(x)$, интерполирующую в точках сетки $\Delta_{p}$ произвольную последовательность $y \in Y_{3}$. Для этого наряду с исходной сеткой (2.1) рассмотрим еще одну сетку узлов $\tilde{\Delta}_{p}=\left\{t_{k}\right\}_{k=-\infty}^{+\infty}$, где $t_{k}=\left(x_{k+1}-x_{k}\right) r+x_{k}, r=\left(p^{2}-\sqrt{p\left(p^{2}-p+1\right)}\right)\left(p^{2}+1\right)^{-1}$. 
Нетрудно видеть, что при всех $p>1$ имеет место неравенство $0<r<1$, и потому $x_{k}<t_{k}<x_{k+1}$ для всех $k \in \mathbb{Z}$, т. е. узлы этих сеток строго чередуются. При $p=1$ имеем $r=0$ и $t_{k}=x_{k}(k \in \mathbb{Z})$, что согласуется с выбором узлов интерполяционных сплайнов нечетной степени (см., например, [6, гл. 2]).

Следуя подходу Ю. Н. Субботина [3], полагаем

$$
\tilde{f}^{\prime \prime \prime}(x)= \begin{cases}Z_{k}, & x_{k} \leq x<t_{k}, \\ Z_{k+1}, & t_{k} \leq x<t_{k+1}, \\ Z_{k+2}, & t_{k+1} \leq x<t_{k+2}, \\ Z_{k+3}, & t_{k+2} \leq x<x_{k+3},\end{cases}
$$

где $\left\{Z_{k}\right\}_{k=-\infty}^{+\infty}-$ неизвестные действительные числа.

Поскольку $\tilde{f}\left(x_{k}\right)=y_{k}(k \in \mathbb{Z})$, то из (3.1) с помощью леммы 1 получаем

$$
\begin{gathered}
Z_{k+3} \int_{t_{k+2}}^{x_{k+3}}\left(x_{k+3}-t\right)^{2} d t \\
+Z_{k+2}\left(\int_{x_{k+2}}^{t_{k+2}}\left(x_{k+3}-t\right)^{2} d t \int_{t_{k+1}}^{x_{k+2}}\left(\left(x_{k+3}-t\right)^{2}-\left(1+p+p^{2}\right)\left(x_{k+2}-t\right)^{2}\right) d t\right) \\
+\int_{t_{k}}^{t_{k+1}}\left(\int_{x_{k+1}}^{x_{k+1}}\left(\left(x_{k+3}-t\right)^{2}-\left(1+p+p^{2}\right)\left(x_{k+2}-t\right)^{2}\right) d t\right. \\
+Z_{k} \int_{x_{k}}^{t_{k}}\left(\left(x_{k+3}-t\right)^{2}-\left(1+p+p^{2}\right)\left(x_{k+2}-t\right)^{2}+p\left(1+p+p^{2}\right)\left(x_{k+1}-t\right)^{2}\right) d t \\
=2\left[y_{k+3}, y_{k+2}, y_{k+1}, y_{k}\right] p^{3(k+1)}(p-1)\left(p^{2}-1\right)\left(p^{3}-1\right) h^{3} .
\end{gathered}
$$

Вычислив интегралы и воспользовавшись формулами для $x_{k}$ и $t_{k}$, приходим к разностному уравнению относительно неизвестных чисел $\left\{Z_{k}\right\}_{k=-\infty}^{+\infty}$ :

$$
a Z_{k+3}+b Z_{k+2}+c Z_{k+1}+d Z_{k}=6 p^{3}(p+1)\left(1+p+p^{2}\right)\left[y_{k+3}, y_{k+2}, y_{k+1}, y_{k}\right] \quad(k \in \mathbb{Z}),
$$

в котором не зависящие от $k$ коэффициенты $a, b, c, d$ имеют вид

$$
\begin{gathered}
a=a(p)=p^{6}(1-r)^{3}, \\
b=b(p)=p^{3}\left[(p+1-r)^{3}-\left(1+p+p^{2}+p^{3}\right)(1-r)^{3}\right], \\
c=c(p)=\left(p^{2}+p+1-r\right)^{3}-\left(p^{3}+p^{2}+p+1\right)(p+1-r)^{3}+\left(p^{5}+p^{4}+2 p^{3}+p^{2}+p\right)(1-r)^{3}, \\
d=d(p)=p^{3}(p+1)\left(1+p+p^{2}\right)-\left(p^{2}+p+1-r\right)^{3}+\left(1+p+p^{2}\right)(p+1-r)^{3}-\left(p+p^{2}+p^{3}\right)(1-r)^{3} .
\end{gathered}
$$

Для исследования разностного уравнения (3.2), (3.3) нам потребуются

Теорема А. Если все корни полинома с действительными коэбфициентами

$$
P_{n}(z)=\sum_{\nu=0}^{n} c_{\nu} z^{\nu}\left(c_{n} \neq 0\right)
$$


являются отрицательными, простыми и $P_{n}(-1) \neq 0$, то разностное уравнение

$$
\sum_{\nu=0}^{n} c_{\nu} Z_{k+\nu}=M_{k} \quad(k \in \mathbb{Z})
$$

где $M=\left\{M_{k}\right\}_{k=-\infty}^{+\infty} \in l_{q}(1 \leq q \leq \infty)$, имеет единственное решение $Z^{0}=\left\{Z_{k}^{0}\right\}_{k=-\infty}^{+\infty} \in l_{q}$,

$$
Z_{k}^{0}=\sum_{s=-\infty}^{+\infty} a_{-s-k} M_{s}, \quad \sum_{s=-\infty}^{+\infty} a_{s} z^{s}=\left(P_{n}(z)\right)^{-1},
$$

и для решения справедлива оченка

$$
\left\|Z^{0}\right\|_{l_{q}} \leq \frac{\|M\|_{l_{q}}}{\left|P_{n}(-1)\right|}
$$

В теореме А существование и единственность решения были доказаны М. Г. Крейном [7], а оценка для $\left\|Z^{0}\right\|_{l_{q}}$ получена Ю.Н. Субботиным [8, с. 124, 125] (см. также с. 203 в обзоре авторов 2018 г.)

Обозначим правую часть уравнения (3.2) через $M_{k}$, т. е.

$$
M_{k}=6 p^{3}(p+1)\left(1+p+p^{2}\right)\left[y_{k+3}, y_{k+2}, y_{k+1}, y_{k}\right] .
$$

Замечаем, что последовательность $\left\{M_{k}\right\}_{k=-\infty}^{+\infty}$ ограничена, поскольку согласно определению класса $Y_{3}$ для любого целого числа $k$ имеет место неравенство $\left|\left[y_{k+3}, y_{k+2}, y_{k+1}, y_{k}\right]\right| \leq 1$.

Через $P(z)=a z^{3}+b z^{2}+c z+d$ всюду далее будем обозначать характеристический полином уравнения (3.2). Для того чтобы применить теорему А к разностному уравнению (3.2), (3.3), нужно исследовать полином $P(z)$, доказать, что все его корни отрицательные и простые, вычислить $P(-1)$ и убедиться в том, что $P(-1) \neq 0$. Это будет сделано далее в леммах $2-5$.

Полагаем $\lambda=1-r$ и переписываем выражения для коэффициентов (3.3) в терминах параметра $\lambda$. После выполнения элементарных преобразований имеем

$$
\begin{gathered}
a=p^{6} \lambda^{3}, \quad b=p^{4}\left(-\lambda^{3}\left(1+p+p^{2}\right)+3 \lambda^{2}+3 p \lambda+p^{2}\right), \\
c=p^{3}\left[\left(1+p+p^{2}\right) \lambda^{3}-3(p+1) \lambda^{2}-3\left(p^{2}-1\right) \lambda+2 p(p+1)\right], \quad d=p^{3}(1-\lambda)^{3} .
\end{gathered}
$$

Лемма 2. При всех $p>1$ справедливы неравенства $a=a(p)>0, b=b(p)>0$, $c=c(p)>0, d=d(p)>0$.

Д о к а з а т ел ь с т в о. Поскольку

$$
\lambda=1-r=\frac{1+\sqrt{p\left(p^{2}-p+1\right)}}{1+p^{2}},
$$

то $0<\lambda<1$ при всех $p>1$. Отсюда сразу же получаем, что $a(p)>0, d(p)>0$.

Подставив $\lambda$ в выражение для $b(p)$, после выполнения несложных преобразований имеем $b(p)=\frac{p^{4}}{\left(1+p^{2}\right)^{3}}\left[p^{8}+3 p^{6}+3 p^{5}+9 p^{3}+2 p+2+\sqrt{p\left(p^{2}-p+1\right)}(p+1)\left(2 p^{4}-2 p^{3}+7 p^{2}-4 p+3\right)\right]$.

Так как $2 p^{4}-2 p^{3}+7 p^{2}-4 p+3=2 p^{3}(p-1)+\left(7 p^{2}-4 p+3\right)$ и дискриминант квадратного трехчлена $7 p^{2}-4 p+3$ отрицателен, то $b(p)>0$.

Подставив $\lambda$ в выражение для $c(p)$, получаем

$$
c(p)=\frac{p^{3}}{\left(1+p^{2}\right)^{3}}\left[2 p^{8}+2 p^{7}+9 p^{5}+3 p^{3}+3 p^{2}+1-p(p+1) \sqrt{p\left(p^{2}-p+1\right)} q(p)\right],
$$


где $q(p)=3 p^{4}-4 p^{3}+7 p^{2}-2 p+2$.

Исследуем знак полинома $q(p)$. Так как

$$
q^{\prime}(p)=12 p^{3}-12 p^{2}+14 p-2=\left(12 p^{2}+2\right)(p-1)+12 p>0,
$$

то полином $q(p)$ возрастает при $p \geq 1$. Поскольку $q(1)>0$, отсюда заключаем, что $q(p)>0$.

Теперь в неравенстве $\sqrt{\alpha \beta} \leq(\alpha+\beta) / 2,(\alpha, \beta \geq 0)$ полагаем $\alpha=p^{3}, \beta=p^{2}-p+1$ и в результате имеем $p \sqrt{p\left(p^{2}-p+1\right)} \leq\left(p^{3}+p^{2}-p+1\right) / 2$. Воспользовавшись этим неравенством, после выполнения элементарных преобразований получаем

$$
\begin{gathered}
c(p) \geq \frac{p^{3}}{\left(1+p^{2}\right)^{3}}\left[1+3 p^{2}+3 p^{3}+9 p^{5}+2 p^{7}+2 p^{8}-(p+1)\left(3 p^{4}-4 p^{3}+7 p^{2}-2 p-2\right)\left(\frac{p^{3}+p^{2}-p+1}{2}\right)\right] \\
\quad=\frac{p^{3}\left(p^{8}+2 p^{7}+p^{6}+6 p^{5}-p^{4}+6 p^{3}-p^{2}+2 p\right)}{2\left(1+p^{2}\right)^{3}}=\frac{p^{4}\left(p^{5}(p+1)^{2}+p\left(p^{2}+1\right)(6 p-1)+2\right)}{2\left(1+p^{2}\right)^{3}}>0 .
\end{gathered}
$$

Лемма 2 доказана.

3 а м е ч а н и е 1 . При $p=1$ имеем $a=a(1)>0, b=b(1)>0, c=c(1)>0, d=d(1)=0$.

Лемма 3. Справедливы равенства

$$
P(-1)=-a+b-c+d=\frac{p^{3}(p+1)\left(p^{2}-p+1\right)}{\left(1+p^{2}\right)^{2}}\left[(p-1)^{3}(p+1)+4 p \sqrt{p\left(p^{2}-p+1\right)}\right] .
$$

Д о к а з а т е л ь с т в о. Обозначаем $S=-a+b-c+d$. Из (3.4) и (3.5) после выполнения элементарных преобразований приходим к выражению

$$
\begin{gathered}
S=-\frac{\left(1+\sqrt{p\left(p^{2}-p+1\right)}\right)^{3}}{\left(1+p^{2}\right)^{3}}\left(2 p^{6}+2 p^{5}+2 p^{4}+4 p^{3}+2 p^{2}+2 p\right) \\
+2\left(p^{3}+p^{2}+p+1\right) \frac{\left(p^{3}+p+1+\sqrt{p\left(p^{2}-p+1\right)}\right)^{3}}{\left(1+p^{2}\right)^{3}} \\
-2 \frac{\left(p^{4}+p^{3}+p^{2}+p+1+\sqrt{p\left(p^{2}-p+1\right)}\right)^{3}}{\left(1+p^{2}\right)^{3}}+p^{3}\left(p^{2}+p+1\right)(p+1) .
\end{gathered}
$$

Обозначив $v=\sqrt{p\left(p^{2}-p+1\right)}$, переписываем полученное выражение в виде

$$
S=\frac{1}{\left(1+p^{2}\right)^{3}}\left[-2 p^{3}(1+p)\left(1+p^{2}\right) v^{3}+6 p^{4}\left(1+p^{2}\right)\left(1+p^{3}\right) v+Q_{12}(p)\right]
$$

где $Q_{12}(p)=p^{12}-2 p^{11}+p^{10}+p^{9}-3 p^{8}+3 p^{7}-p^{6}-p^{5}+2 p^{4}-p^{3}-$ алгебраический полином степени 12 от переменной $p$. Замечаем, что этот полином имеет корни: $p=0$ и $p=1$ третьей кратности, $p=-1$ второй кратности и $p= \pm i$ ( $i$ - мнимая единица) первой кратности. Поэтому

$$
Q_{12}(p)=p^{3}\left(p^{2}+1\right)(p+1)^{2}(p-1)^{3}\left(p^{2}-p+1\right)
$$

и

$$
\begin{aligned}
S= & \frac{(p+1) p^{3}}{\left(1+p^{2}\right)^{2}}\left[-2 v^{3}+6 p\left(p^{2}-p+1\right) v+(p+1)(p-1)^{3}\left(p^{2}-p+1\right)\right] \\
= & \frac{p^{3}(p+1)\left(p^{2}-p+1\right)}{\left(1+p^{2}\right)^{2}}\left[(p-1)^{3}(p+1)+4 p \sqrt{p\left(p^{2}-p+1\right)}\right] .
\end{aligned}
$$

Лемма 3 доказана. 
Лемма 4. При всех $p \geq 2$ выполняется неравенство

$$
P\left(-\frac{1}{p^{2}}\right)<0
$$

Д о к а з а т е л ь с т в о. Подставив в выражение

$$
P\left(-\frac{1}{p^{2}}\right)=-a \frac{1}{p^{6}}+b \frac{1}{p^{4}}-c \frac{1}{p^{2}}+d
$$

значения коэффициентов $a, b, c, d$ из (3.4), получаем

$$
P\left(-\frac{1}{p^{2}}\right)=-(p+1)\left(2 \lambda^{3}\left(1+p^{2}\right)-3 \lambda^{2}\left(1+p^{2}\right)+p^{2}\right) .
$$

Достаточно установить, что при $p \geq 2$ выполняется неравенство

$$
2 \lambda^{3}\left(1+p^{2}\right)-3 \lambda^{2}\left(1+p^{2}\right)+p^{2}>0 .
$$

Заменяя $\lambda$ его значением из (3.5), после выполнения несложных вычислений имеем

$$
2 \lambda^{3}\left(1+p^{2}\right)-3 \lambda^{2}\left(1+p^{2}\right)+p^{2}=\frac{1}{\left(1+p^{2}\right)^{2}} G(p),
$$

где

$$
\begin{aligned}
& G(p)=p^{6}-3 p^{5}+5 p^{4}-5 p^{2}+3 p-1+2 p\left(p^{2}-4 p+1\right) \sqrt{p\left(p^{2}-p+1\right)} \\
& =\left(p^{2}-1\right)\left(p^{4}-3 p^{3}+6 p^{2}-3 p+1\right)+2 p\left(p^{2}-4 p+1\right) \sqrt{p\left(p^{2}-p+1\right)} .
\end{aligned}
$$

Далее замечаем, что $G(2)=33-12 \sqrt{6}>0$, и при $p=2$ лемма 4 доказана.

Поскольку выражение $p^{2}-4 p+1$ меняет знак в точке $p=2+\sqrt{3}$, то дальнейшее доказательство при $p>2$ разбивается на два случая.

1) Пусть $p \geq 2+\sqrt{3}$. Воспользовавшись неравенством

$$
\sqrt{p\left(p^{2}-p+1\right)} \geq \sqrt{p\left(p^{2}-2 p+1\right)}=\sqrt{p}(p-1)>p-1,
$$

имеем

$$
\begin{aligned}
& G(p)>\left(p^{2}-1\right)\left(p^{4}-3 p^{3}+6 p^{2}-3 p+1\right)+2 p\left(p^{2}-4 p+1\right)(p-1) \\
= & (p-1)^{2}\left(p^{4}-p^{3}+4 p^{2}-p+1\right)=(p-1)^{2}\left[p^{3}(p-1)+\left(4 p^{2}-p+1\right)\right]>0,
\end{aligned}
$$

поскольку $4 p^{2}-p+1>0$.

2) Пусть теперь $2<p<2+\sqrt{3}$. Как и при доказательстве леммы 2 , в неравенстве $\sqrt{\alpha \beta} \leq(\alpha+\beta) / 2$ полагаем $\alpha=p^{3}, \beta=p^{2}-p+1$ и в результате имеем

$$
2 p \sqrt{p\left(p^{2}-p+1\right)} \leq p^{3}+p^{2}-p+1 .
$$

Применив это неравенство к выражению для $G(p)$, получаем

$$
\begin{aligned}
G(p) & \geq\left(p^{2}-1\right)\left(p^{4}-3 p^{3}+6 p^{2}-3 p+1\right)+\left(p^{2}-4 p+1\right)\left(p^{3}+p^{2}-p+1\right) \\
& =p\left(p^{5}-2 p^{4}+2 p^{3}-4 p^{2}+p-2\right)=p\left(p^{2}+1\right)^{2}(p-2) .
\end{aligned}
$$

Поскольку $p>2$, то $G(p)>0$, и лемма 4 полностью доказана.

Лемма 5. При всех $1 \leq p<2$ справедливо неравенство

$$
P\left(-\frac{1}{4 p}\right)<0
$$


Д о к а з а т е л ь с т в о. Пусть $1 \leq p<2$. Подставив $z=-1 / 4 p$ в $P(z)$ и воспользовавшись равенствами (3.4), имеем

$$
\begin{gathered}
P\left(-\frac{1}{4 p}\right)=-\frac{a}{64 p^{3}}+\frac{b}{16 p^{2}}-\frac{c}{4 p}+d \\
=-\frac{p^{2}}{64}\left[5(p+4)(4 p+1) \lambda^{3}-60(4 p+1) \lambda^{2}-12(p-4)(4 p+1) \lambda+4 p(7 p-8)\right] .
\end{gathered}
$$

Заменяем $\lambda$ его выражением через $p$ (см. (3.5)) и выполняем элементарные преобразования. В результате получаем

$$
P\left(-\frac{1}{4 p}\right)=-\frac{p^{2}}{64\left(1+p^{2}\right)^{3}}\left(q_{1}(p)+\sqrt{p\left(p^{2}-p+1\right)} q_{2}(p)\right),
$$

где

$$
\begin{aligned}
& q_{1}(p)=28 p^{8}-32 p^{7}-204 p^{6}+324 p^{5}-189 p^{4}+9 p^{3}+51 p^{2}-7 p+8, \\
& q_{2}(p)=-48 p^{6}+200 p^{5}+17 p^{4}-165 p^{3}+53 p^{2}-25 p-12 .
\end{aligned}
$$

Достаточно доказать, что $q_{1}(p)+\sqrt{p\left(p^{2}-p+1\right)} q_{2}(p)>0$.

Прежде всего покажем, что $q_{2}(p)>0$. Замечаем, что полином $q_{2}(p)$ имеет нули в точках $p=-1, p=-1 / 4$, и потому

$$
q_{2}(p)=-(p+1)(4 p+1) \tau(p),
$$

где $\tau(p)=12 p^{4}-65 p^{3}+74 p^{2}-35 p+12$. Следовательно, остается убедиться в том, что $\tau(p)<0$ при $1 \leq p<2$.

Поскольку $\tau^{\prime \prime}(p)=2\left(72 p^{2}-195 p+74\right)$ и квадратный трехчлен имеет два вещественных корня, один из которых больше двух, а другой лежит в интервале $(0,1)$, то $\tau^{\prime \prime}(p)<0$ при $1 \leq p<2$. Поэтому $\tau^{\prime}(p)$ при всех $1 \leq p<2$ убывает, а так как $\tau^{\prime}(1)<0$, то $\tau^{\prime}(p)<0$ на $[1,2)$. Следовательно, полином $\tau(p)$ также убывает, и поскольку $\tau(1)=-2<0$, то он отрицателен и потому $q_{2}(p)>0$.

Нетрудно видеть, что $\sqrt{p\left(p^{2}-p+1\right)} \geq p$ при всех $p \geq 1$. Таким образом,

$$
q_{1}(p)+\sqrt{p\left(p^{2}-p+1\right)} q_{2}(p) \geq q_{1}(p)+p q_{2}(p)=V(p),
$$

где $V(p)=28 p^{8}-80 p^{7}-4 p^{6}+341 p^{5}-354 p^{4}+62 p^{3}+26 p^{2}-19 p+8$.

Перепишем полином $V(p)$ в виде

$$
V(p)=8+p(p-1) c_{1}+p(p-1)^{2} c_{2}+p(p-1)^{3} Q(p),
$$

где $Q(p)=28 p^{4}+4 p^{3}-76 p^{2}+129 p+265, c_{1}=148, c_{2}=394$, и докажем, что при всех $p \in[1,2)$ выполняется неравенство $Q(p)>0$.

Так как $Q^{\prime \prime}(p)=8\left(42 p^{2}+3 p-12\right)$ и квадратный трехчлен имеет два вещественных корня, один из которых отрицателен, а другой принадлежит интервалу $(0,1)$, то $Q^{\prime \prime}(p)>0$ при всех $p \geq 1$. Поэтому $Q^{\prime}(p)$ возрастает, а поскольку $Q^{\prime}(1)>0$, то $Q^{\prime}(p)>0$ при $p \geq 1$. Следовательно, полином $Q(p)$ также возрастает и благодаря тому, что $Q(1)>0$, приходим к неравенству $Q(p)>0$.

Теперь из представления (3.7) при всех $p \geq 1$ имеем

$$
V(p)>p(p-1)^{3} Q(p) \geq 0
$$

и, возвращаясь к равенству (3.6), получаем $P(-1 / 4 p)<0$. Лемма 5 доказана.

Переходим к получению оценки сверху $L_{\infty}$-нормы третьей производной построенного интерполянта. 
Утверждение 2. Пусть у $\in Y_{3}$ - произвольная последовательность. Тогда при всех $p>1$ для функиии $\tilde{f} \in F_{3}(y)$ справедливо неравенство

$$
\sup _{x \in \mathbb{R}_{+}}\left|\tilde{f}^{\prime \prime \prime}(x)\right| \leq \frac{6\left(p^{2}+p+1\right)}{\left(p^{2}-p+1\right)} \frac{\left(p^{2}+1\right)^{2}}{(p-1)^{3}(p+1)+4 p \sqrt{p\left(p^{2}-p+1\right)}} .
$$

Д о к а з а т е л ь с т в о. Из построения функции $\tilde{f}(x)$ имеем

$$
\sup _{x \in \mathbb{R}_{+}}\left|\tilde{f}^{\prime \prime \prime}(x)\right|=\sup _{k \in \mathbb{Z}}\left|Z_{k}\right|
$$

и числа $\left\{Z_{k}\right\}_{k=-\infty}^{+\infty}$ Удовлетворяют разностному уравнению (3.2), (3.3). Убедимся, что к нему можно применить теорему А.

Из леммы 2 вытекает, что при $p>1$ характеристический полином $P(z)$ уравнения $(3.2)$ не имеет корней на полуоси $[0,+\infty)$. Следовательно, либо все три его корня (с учетом кратностей) являются отрицательными, либо один из корней отрицательный, а два других - комплексно сопряженные с ненулевыми мнимыми частями. Покажем, что реализуется первая из этих двух возможностей, и все корни являются простыми.

Действительно, из леммы 2 имеем $P(0)=d>0$, а из леммы 3 легко видеть, что $P(-1)>0$. При $p \geq 2$ согласно лемме 4 выполняется неравенство $P\left(-1 / p^{2}\right)<0$, а при $1 \leq p<2$ согласно лемме 5 справедливо неравенство $P(-1 / 4 p)<0$, и поскольку $\lim _{z \rightarrow-\infty} P(z)=-\infty$, то при всех $p>1$ полином $P(z)$ имеет три перемены знака на полуоси $(-\infty, 0)$. Отсюда следует, что полином $P(z)$ имеет три различных отрицательных корня.

$\mathrm{K}$ разностному уравнению (3.2) применяем теорему А при $n=3, q=\infty$ и получаем, что оно имеет единственное ограниченное решение $Z^{0}=\left\{Z_{k}^{0}\right\}_{k=-\infty}^{+\infty}$, и для этого решения выполняется оценка

$$
\sup _{k \in \mathbb{Z}}\left|Z_{k}^{0}\right| \leq \frac{6\left(p^{2}+p+1\right)}{\left(p^{2}-p+1\right)} \frac{\left(p^{2}+1\right)^{2}}{(p-1)^{3}(p+1)+4 p \sqrt{p\left(p^{2}-p+1\right)}} .
$$

Тот факт, что функция $\tilde{f}(x)$ принадлежит классу $F_{3}(y)$, доказывается в точности так же, как был установлен аналогичный результат в [3, с. 33] для равномерной сетки точек интерполяции.

Тем самым утверждение 2 доказано.

3 а м е ч а н и е 2 . Функция $\tilde{f} \in F_{3}(y)$ является кубическим сплайном минимального дефекта с узлами "склейки" в точках сетки $\tilde{\Delta}_{p}$ и интерполяцией в точках $\Delta_{p}$.

Д о к а з а т е л ь с т в о теоремы 1. Пусть $p>1$. Для доказательства оценки снизу величины $A_{3}\left(\Delta_{p}\right)$ воспользуемся утверждением 1 , а доказательства оценки сверху - утверждением 2. В результате получаем

$$
\begin{gathered}
A_{3}\left(\Delta_{p}\right) \geq \inf _{f \in F_{3}\left(y^{*}\right)}\left\|f^{\prime \prime \prime}\right\|_{\infty} \geq \frac{6\left(p^{2}+p+1\right)}{\left(p^{2}-p+1\right)} \frac{\left(p^{2}+1\right)^{2}}{(p-1)^{3}(p+1)+4 p \sqrt{p\left(p^{2}-p+1\right)}}, \\
A_{3}\left(\Delta_{p}\right) \leq\left\|\tilde{f}^{\prime \prime \prime}\right\|_{\infty} \leq \frac{6\left(p^{2}+p+1\right)}{\left(p^{2}-p+1\right)} \frac{\left(p^{2}+1\right)^{2}}{(p-1)^{3}(p+1)+4 p \sqrt{p\left(p^{2}-p+1\right)}}
\end{gathered}
$$

Поскольку правые части этих неравенств совпадают, теорема 1 доказана.

Возникает естественный вопрос: при каких значениях $p \in[1,+\infty)$ величина $A_{3}\left(\Delta_{p}\right)$ принимает наименьшее значение? Ответ на него дает

Следствие. Имеет место соотношение

$$
\inf _{p \in[1,+\infty)} A_{3}\left(\Delta_{p}\right)=6
$$

в котором точная нижняя грань достигается при $p \rightarrow+\infty$. 
Д о к а з а т е л ь с т в о. Замечаем, что $\lim _{p \rightarrow+\infty} A_{3}\left(\Delta_{p}\right)=6$. С другой стороны, нетрудно доказать, что при всех $p \geq 1$ выполняется неравенство $A_{3}\left(\Delta_{p}\right)>6$.

Действительно, неравенство

$$
\frac{p^{2}+p+1}{p^{2}-p+1}>1
$$

при положительных $p$ очевидно, а неравенство

$$
\left(p^{2}+1\right)^{2}-(p-1)^{3}(p+1) \geq 4 p \sqrt{p\left(p^{2}-p+1\right)}
$$

эквивалентно неравенству $\alpha+\beta \geq 2 \sqrt{\alpha \beta}$, если в нем положить $\alpha=p^{3}, \beta=p^{2}-p+1$. Отсюда вытекает, что при $p \geq 1$ выполняется $A_{3}\left(\Delta_{p}\right)>6$. Следствие доказано.

\section{Заключение}

Таким образом, для третьей производной найдено точное решение задачи экстремальной функциональной интерполяции в случае интерполяции на неравномерной геометрической сетке. Получение основного результата (теоремы 1) стало возможным благодаря тому, что для геометрической сетки узлов разностное уравнение (3.2) оказалось уравнением с постоянными (а не переменными) коэффициентами, теория решения которых была ранее развита в работах М. Г. Крейна [7] и Ю. Н. Субботина [8].

Остается открытым вопрос: верно ли, что при $n \geq 3$ для любой сетки точек интерполяции $\Delta=\left\{x_{k}\right\}_{k=-\infty}^{+\infty}$ величина $A_{n}(\Delta)$ является конечной?

\section{СПИСОК ЛИТЕРАТУРЫ}

1. Гельфонд А.О. Исчисление конечных разностей. М.: Наука, 1967. 376 с.

2. Favard J. Sur l'interpolation // J. Math. Pures Appl. 1940. Vol. 19. P. 281-306.

3. Субботин Ю.Н. О связи между конечными разностями и соответствующими производными // Тр. МИАН СССР. 1965. Т. 78. С. 24-42.

4. Субботин Ю.Н. Функциональная интерполяция в среднем с наименьшей $n$-й производной $/ /$ Тр. МИАН СССР. 1967. Т. 88. С. 30-60.

5. Kunkle Th. Favard's interpolation problem in one or more variables // Constructive Approxim. 2002. Vol. 18. P. 467-478. doi: 10.1007/s00365-001-0015-7.

6. Алберг Дж,, Нильсон Э., Уолш Дж. Теория сплайнов и ее приложения М.: Мир, 1972. 316 с.

7. Крейн М.Г. Интегральные уравнения на полупрямой с ядрами, зависящими от разности аргументов // Успехи матем. наук. 1958. Т. 13, № 5 (83). С. 3-120.

8. Субботин Ю.Н. Экстремальные задачи функциональной интерполяции и интерполяционные в среднем сплайны // Тр. МИАН СССР. 1975. Т. 138. С. 118-173.

Поступила 9.09.2020

После доработки 23.10.2020

Принята к публикации 2.11.2020

Новиков Сергей Игоревич

канд. физ.-мат. наук

старший научный сотрудник

Институт математики и механики им. Н. Н. Красовского УрО РАН

г. Екатеринбург

e-mail: Sergey.Novikov@imm.uran.ru

Шевалдин Валерий Трифонович

д-р физ.-мат. наук

ведущий научный сотрудник

Институт математики и механики им. Н. Н. Красовского УрО РАН

г. Екатеринбург

e-mail: Valerii.Shevaldin@imm.uran.ru 


\section{REFERENCES}

1. Gel'fond A.O. Calculus of finite differences. Delhi: Hindustan Publ. Corp., 1971, ser. International Monographs on Advanced Mathematics and Physics, 451 p. Original Russian text published in Gel'fond A.O. Ischislenie konechnykh raznostey. Moscow: Nauka Publ., 1967.

2. Favard J. Sur l'interpolation. J. Math. Pures Appl., 1940, vol. 19, no. 9, pp. 281-306.

3. Subbotin Yu.N. On the connection between finite differences and corresponding derivatives. Proc. Steklov Inst. Math., 1965, vol. 78, pp. 24-42 (in Russian).

4. Subbotin Yu.N. Functional interpolation in the mean with smallest $n$ derivative. Proc. Steklov Inst. Math., 1967, vol. 88, pp. 31-63.

5. Kunkle Th. Favard's interpolation problem in one or more variables. Constructive Approxim., 2002, vol. 18, pp. 467-478. doi: 10.1007/s00365-001-0015-7.

6. Ahlberg J., Nilson E., Walsh J. The theory of splines and their applications. N Y: Acad. Press, 1967, 284 p. ISBN: 9781483222950 . Translated to Russian under the title Teoriya splainov i ee prilozheniya. Moscow: Mir Publ., 1972, 316 p.

7. Krein M.G. Integral equations on the half-line with a kernel depending on the difference of the arguments. Uspekhi Mat. Nauk, 1958, vol. 13, no. 5(83), pp. 3-120 (in Russian).

8. Subbotin Yu.N. Extremal problems of functional interpolation, and mean interpolation splines. Proc. Steklov Inst. Math., 1977, vol. 138, pp. 127-185.

Received September 30, 2020

Revised October 23, 2020

Accepted November 2, 2020

Funding Agency: This study is a part of the research carried out at the Ural Mathematical Center.

Sergey Igorevich Novikov, Cand. Sci. (Phys.-Math.), Krasovskii Institute of Mathematics and Mechanics of the Ural Branch of the Russian Academy of Sciences, Yekaterinburg, 620108 Russia, e-mail: Sergey.Novikov@imm.uran.ru .

Valerii Trifonovich Shevaldin, Dr. Phys.-Math. Sci., Krasovskii Institute of Mathematics and Mechanics Ural Branch of the Russian Academy of Sciences, Yekaterinburg, 620108 Russia, e-mail: Valerii.Shevaldin@imm.uran.ru .

Cite this article as: S. I. Novikov, V. T. Shevaldin. Extremal interpolation on the semiaxis with the smallest norm of the third derivative, Trudy Instituta Matematiki i Mekhaniki UrO RAN, 2020, vol. 26 , no. 4 , pp. 210-223. 